\title{
Spectrum Sensing and Data Transmission in a Cognitive Relay Network Considering Spatial False Alarms
}

\author{
Tasnina A. Tishita*, Sumiya Akhter*, Md. Imdadul Islam**, and M. R. Amin*
}

\begin{abstract}
In this paper, the average probability of the symbol error rate (SER) and throughput are studied in the presence of joint spectrum sensing and data transmission in a cognitive relay network, which is in the environment of an optimal power allocation strategy. In this investigation, the main component in calculating the secondary throughput is the inclusion of the spatial false alarms, in addition to the conventional false alarms. It has been shown that there exists an optimal secondary power amplification factor at which the probability of SER has a minimum value, whereas the throughput has a maximum value. We performed a Monte-Carlo simulation to validate the analytical results.
\end{abstract}

Keywords-Cognitive Network, Conventional False Alarms, Probability of Symbol Error Rate, Spatial False Alarms, Spectrum Sensing

\section{INTRODUCTION}

With the explosive growth of wireless communication systems, such as wireless local area networks (WLANs), mobile cellular networks, ad-hoc and sensor networks, etc., the demand for the radio spectrum has rapidly increased and thus, the wireless spectrum has become a scarce commodity. The traditional schemes of fixed spectrum allocation have been shown to be inadequate in addressing such quickly evolving wireless technologies. Recent measurement studies [1] have demonstrated that many frequency bands licensed to operators are employed in scattered fashion over vast geographical areas and are idle most of the time (commonly known as spectrum holes). Cognitive radio (CR) systems have been proposed as a promising solution to inefficient spectrum management and scarcity of the radio spectrum. It is defined as an intelligent wireless communication system [2] that is aware of the surrounding environment and utilizes the methodology of understanding-by-building to learn from the environment. The spectrum detection technique (also referred to as spectrum sensing) enables CR networks to adapt to the environment by detecting spectrum holes. The most efficient way to detect the spectrum holes is to detect the presence of primary users (PUs) $[3,4]$. However, in reality, it is difficult for a $\mathrm{CR}$ to obtain a direct measurement of the channel between a primary receiver and a transmitter. Therefore, the most recent work focuses on the primary transmitter detection method, which is based on the local observations of secondary users (SUs).

Starting from the idea of enabling devices to use any available spectrum $[5,6]$, the concept of

Manuscript received May 22, 2013; accepted July 21, 2013; onlinefirst August 25, 2014

Corresponding Author: M. Ruhul Amin (ramin@ewubd.edu)

* Department of Electronics and Communications Engineering, East West University, Aftabnagar, Dhaka 1212, Bangladesh (tishita_272@yahoo.com, sumiya.choity@yahoo.com,ramin@ewubd.edu)

** Department of Computer Science and Engineering, Jahangirnagar University, Savar, Dhaka 1342, Bangladesh (imdad@juniv.edu) 
$\mathrm{CR}$ has been well acceptedwithin the wireless communications research community. The key idea is to use portions of the spectrum that are not being used by the licensed PUs for the benefit of the unlicensed SUs and to do so without causing harmful interference to the PUs.

The fundamental concept of the CR was first introduced by Mitola [5]. In a CR system, the $\mathrm{SU}$, sometimes called a cognitive user (CU), should be able to recognize the radio environment to accurately search for spectrum holes that are not exploited by the PUs. In this system, the PUs should be sufficiently protected. Thus, spectrum sensing in the secondary network is one of the most important issues [3,4,7].

In [8], the authors have developed a theory to calculate the secondary throughput in a CR relay network system under the optimal power allocation (OPA) scheme. The total power transmitted by the cognitive sourceand the cognitive relay is assumed to be constant. In this analysis, the authors have shown that there exists an optimal amplifying gain to achieve maximum secondary throughput under certain conditions. It is also shown that compared to equal power allocation, the throughput gain by OPA increases with the total budget and the OPA always outperforms the equal power allocation.

On the other hand in [9], the authors have developed a theory to show that apart from the conventional false alarm (CFA) probability, there can always be an access opportunity loss due to not properly considering the spectrum sensing in the CR system. The issue is that with a certain probability, a SU misinterprets a non-interfered PU as being interfered with. This then results in an access opportunity loss. This issue is known as a spatial false alarm (SFA) problem and is one of determinants in the SU's medium access probability [10-13]. So far, this issue has been neglected in current research related to spectrum sensing. The authors of [9] have presented in detail the principle of SFA and have quantified the impact of SFA by deriving a closed form expression to correctly evaluate the medium access probability for the SU.

In [8], only the CFA is considered in calculating the secondary throughput. However, for actual quantification of the secondary medium access probability, the effect of the SFA probability has to be included [9]. In our paper, we have extended the work of Huang et al. [8] to calculate the secondary throughput under the OPA scheme. We did so by including the impact of the SFA probability in addition to the CFA probability. We also calculated the average probability of symbol error rate (SER) for different fading channels (viz. the Rayleigh and Nakagami- $m$ fading channels) to get the real scenario of a CR network in an urban area. In order to optimize the performance of the network, we also investigated the impact of the fading parameters and the sensing range on the profile of the probability of correct decision.

The rest of the paper is organized as follows: the system and channel model is described in Section 2. In this section, expressions for the average probability of SER and throughput are presented. Results are discussed in Section 3 and graphical representations are presented. Finally, we make some concluding remarks in Section 4.

\section{SYSTEM DESCRIPTION}

As in [8], we considered a CR system consisting of a PU and a three-node cognitive relay network (CRN) (i.e., a cognitive source [CS], a CR, and a cognitive destination [CD]). The total transmission power of the $\mathrm{CRN}$ is assumed to be fixed. Thus, with a fixed cognitive transmission power, if more power is allocated to the CS for data transmission, the CR will have 
less power for spectrum sensing, which may lead to worse sensing performance. On the other hand, the sensing performance must be satisfied in order to restrict the interference of the CS with the PU. Therefore, to maximize the secondary throughput, an OPA strategy is needed for the secondary network.

For the purpose of quantitative analysis and a practical scenario, let us consider that a PU is located at a distance $R_{0}$ from the SU. We further considered that the PU has the probability $\mathrm{p}$ to be in the transmitting state at any moment in time. The small-scale primary network was considered to be as follows: for the existence of a PU in a circular observed window, of which the area is $\pi R_{0}^{2}$, where $R_{0}>r_{s}$ [9]. Here the spectrum sensing needs to properly determine one of the two states $\mathcal{H}_{0}$ and $\mathcal{H}_{1}$, where $\mathcal{H}_{0}$ denotes that the access opportunity is that will be available; and $\mathcal{H}_{1}$ denotes the access opportunity that will be unavailable for which the PU is in a transmitting state inside the sensing range. In addition, the state $\mathcal{H}_{0}$ is divided into two substates: $\mathcal{H}_{0,-}$ and $\mathcal{H}_{0,+}$. The sub-state $\mathcal{H}_{0,-}$ denotes that the PU is not in the transmitting state and $\mathcal{H}_{0,+}$ denotes that the $\mathrm{PU}$ is in the transmitting state but is residing at a point outside the sensing range.

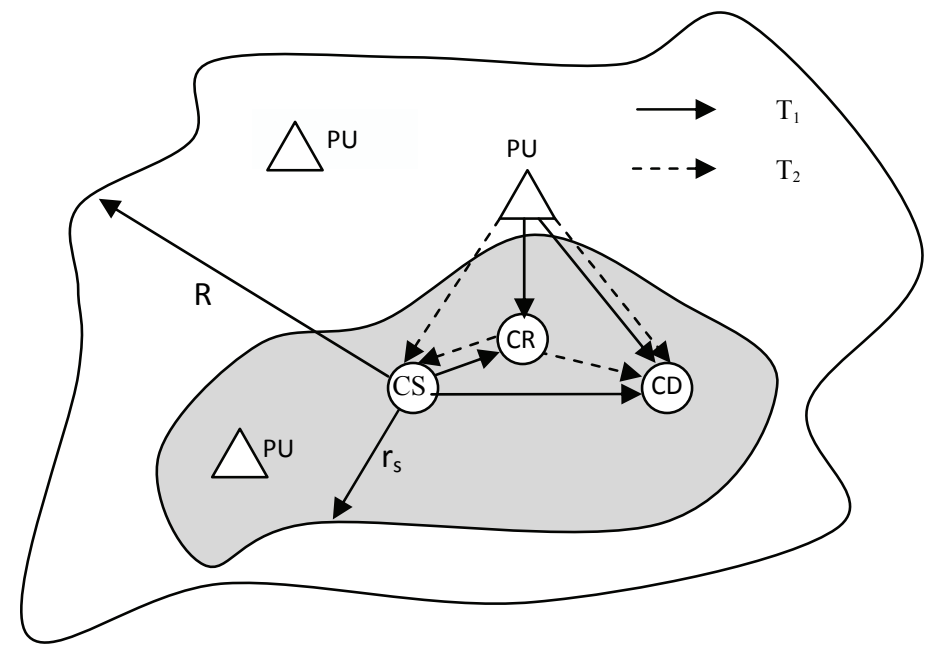

Fig. 1. The cognitive radio system. $P U=$ primary user, $C S=$ cognitive source, $C R=$ cognitive relay, $C D=$ cognitive destination.

The CR system is shown in Fig. 1, which consists of a PU and a CRN with three CUs (the CS, $\mathrm{CR}$, and $\mathrm{CD}$ ). The $\mathrm{CRN}$ operates on a frame-by-frame basis. Each frame is assumed to have two phases $T_{1}$ and $T_{2}\left(T_{1}=T_{2}\right)$. In each frame duration of $\left(T_{1}+T_{2}\right)$, the CRN continues monitoring the presence of the PU. If the PU is not detected in the previous frame, the CRN then transmits data and monitors the presence of the PU simultaneously in the current frame. Otherwise, if the PU is detected in the previous frame, the CRN does not transmit data but only monitors the presence of the PU in the current frame. We need to mention here that in the very first frame, the CRN only monitors the presence of the PU but does not transmit data.

The channels over the links are the following: 
$\mathrm{PU} \rightarrow \mathrm{CS}, \mathrm{PU} \rightarrow \mathrm{CR}, \mathrm{PU} \rightarrow \mathrm{CD}, \mathrm{CS} \leftrightarrow \mathrm{CR}, \mathrm{CR} \rightarrow \mathrm{CD}$, and $\mathrm{CS} \rightarrow \mathrm{CD}$. These are modeled to be either Rayleigh or Nakagami flat fading channels with channel coefficients $h_{P D}$ $h_{P S}, h_{P R}, h_{P D}, h_{S R}, h_{R D}$, and $h_{S D}$, respectively. We assume $h_{P S} \sim C N\left(0, d_{P S}^{-v}\right)$, where $v$ is the path loss exponent, $d_{P S}$ is the normalized distance between the PU and the CS. The other coefficients can be described likewise. We further assume that these channel coefficients are known to the CS and the CR, and are constants during a frame. Let us denote $G_{P S}=\left|h_{P S}\right|^{2}$, $G_{P R}=\left|h_{P R}\right|^{2}, G_{S R}=\left|h_{S R}\right|^{2}, G_{R D}=\left|h_{R D}\right|^{2}$, and $G_{S D}=\left|h_{S D}\right|^{2}$, these are the channel gains. We denote the signals transmitted from the PU and the CS by $x_{P}[n]$ and $x_{C}[n]$, respectively, which have a zero mean and unit variance, (i.e., $E\left[x_{P}[n]\right]=0, E\left[x_{C}[n]\right]=0$ and $E\left[\left|x_{P}[n]\right|^{2}\right]=1, E\left[\left|x_{C}[n]\right|^{2}\right]=1$.)

In the first phase $T_{1}$, the $\mathrm{CR}$ and the $\mathrm{CD}$ listen to the PU while the CS transmits data to the CR and the CD. Thus, the signals received by the $\mathrm{CR}$ and the $\mathrm{CD}$ in $T_{1}$ can be written as [8]:

$$
y_{R 1}[n]=\sqrt{P_{S}} h_{S R} x_{C 1}[n]+\theta \sqrt{P_{P}} h_{P R} x_{P 1}[n]+n_{R 1}[n],
$$

and

$$
y_{D 1}[n]=\sqrt{P_{S}} h_{S D} x_{C 1}[n]+\theta \sqrt{P_{P}} h_{P D} x_{P 1}[n]+n_{D 1}[n],
$$

respectively, where $n=1,2, \cdots, N, N=T_{1} f_{S}, f_{S}$ is the sampling rate, $\theta$ is the primary signal indicator: $\theta=1$ implies the presence of the primary signal. Whereas, $\theta=0$ implies the absence of the primary signal; $x_{C 1}[n]$ and $x_{P 1}[n]$, respectively, denote the signals sent from the $\mathrm{CS}$ and the PU in phase $T_{1}$ and they have a zero mean and unit variance, (i.e., $\mathrm{E}\left[x_{P 1}[n]\right]=0, \mathrm{E}\left[x_{C 1}[n]\right]=0$, and $\left.\mathrm{E}\left[\left|x_{P 1}[n]\right|^{2}\right]=1, \mathrm{E}\left[\left|x_{C 1}[n]\right|^{2}\right]=1\right)$. The additive noises $n_{R 1}[n]$ and $n_{D 1}[n]$ are independent and are identically distributed (iid), circularly symmetric complex Gaussian (CSCG) random sequences with a zero mean and variance $E\left[\left|n_{R 1}[n]\right|^{2}\right]=P_{U}, E\left[\left|n_{D 1}[n]\right|^{2}\right]=P_{U}$.

In the second phase $T_{2}$, the CR amplifies the signal that it received in the first phase $T_{1}$ with an amplifying factor $\sqrt{\beta}$ and forwards this amplified signal to the CS and the CD. Furthermore, the CS and the CD listen to the PU. Therefore, the signals received by the CS and the $\mathrm{CD}$ in the second phase $T_{2}$ can be expressed as [8]:

$$
\begin{aligned}
y_{S 2}[n]= & \sqrt{\beta} h_{S R} y_{R 1}[n]+\theta \sqrt{P_{P}} h_{P S} x_{P 2}[n]+n_{S 2}[n] \\
= & \sqrt{\beta} \sqrt{P_{S}} h_{S R} h_{S R} x_{C 1}[n] \\
& \left.+\theta \sqrt{P_{P}}\left(\sqrt{\beta} h_{S R} h_{P R} x_{P 1}[n]+h_{P S} x_{P 2}[n]\right)+\sqrt{\beta} h_{S R} n_{R 1}[n]+n_{S 2}[n]\right],
\end{aligned}
$$

and 


$$
\begin{aligned}
y_{D 2}[n] & =\sqrt{\beta} h_{R D} y_{R 1}[n]+\theta \sqrt{P_{P}} h_{P D} x_{P 2}[n]+n_{D 2}[n] \\
& =\sqrt{\beta} \sqrt{P_{S}} h_{R D} h_{S R} x_{C 1}[n] \\
& \left.+\theta \sqrt{P_{P}}\left(\sqrt{\beta} h_{R D} h_{P R} x_{P 1}[n]+h_{P D} x_{P 2}[n]\right)+\sqrt{\beta} h_{R D} n_{R 1}[n]+n_{D 2}[n]\right],
\end{aligned}
$$

respectively, where $n=N+1, N+2, \cdots, 2 N$, and Eq. (1) is used for $y_{R 1}[n] ; x_{P 2}[n]$ denotes the signal sent from the PU in phase $T_{2}$. This signal $x_{P 2}[n]$ is also assumed to have a zero mean and unit variance (i.e., $E\left[x_{P 2}[n]\right]=0$ and $E\left[\left|x_{P 2}[n]\right|^{2}\right]=1$ ). The noises $n_{S 2}[n]$ and $n_{D 2}[n]$ in phase $T_{2}$ are also iid, and the CSCG randomly sequences with a zero mean and variance $\mathrm{E}\left[\left|n_{S 2}[n]\right|^{2}\right]=\mathrm{E}\left[\left|n_{D 2}[n]\right|^{2}\right]=P_{U}$.

We note that the first term of Eq. (3) is the self-interference at the CS. After canceling this self-interaction term, the remaining signal at the CS in $T_{2}$ can be written as:

$$
\left.\tilde{y}_{S 2}[n]=\theta \sqrt{P_{P}}\left(\sqrt{\beta} h_{S R} h_{P R} x_{P 1}[n]+h_{P S} x_{P 2}[n]\right)+\sqrt{\beta} h_{S R} n_{R 1}[n]+n_{S 2}[n]\right] .
$$

An energy detector is adopted at the CS for spectrum sensing by utilizing the above signal $\tilde{y}_{S 2}[n]$. The test statistic is then given by:

$$
Y=\sum_{n=N+1}^{2 N}\left|\tilde{y}_{S 2}[n]\right|^{2}
$$

It can be shown that the mean and variance of the energy detector test statistic $Y$ under the hypothesis $\mathcal{H}_{1}(\theta=1)$ are $E\left[Y_{1}\right]=N \mu_{1}$ and $D\left[Y_{1}\right]=N \mu_{1}^{2}$, respectively, where:

$$
\mu_{1}=G_{P S} P_{P}+\beta G_{S R}\left(G_{P R} P_{P}+P_{U}\right)+P_{U}
$$

Similarly, the mean and variance of the test statistic $Y$ under the hypothesis $\mathcal{H}_{0}(\theta=0)$ are shown to be $E\left[Y_{0}\right]=N \mu_{0}$ and $D\left[Y_{0}\right]=N \mu_{0}^{2}$, respectively, where:

$$
\mu_{0}=\beta G_{S R} P_{U}+P_{U}
$$

In deriving the above mean and variance, we have assumed that the signals $\left[x_{P 1}[n]\right]$ and $\left[x_{P 2}[n]\right]$ are CSCG random sequences and that these signals and the noises $n_{R 1}[n]$ and $n_{S 2}[n]$ are pairwise independent.

The received cognitive signal-to-noise ratio (CSNR) $\gamma_{0}$ at the $\mathrm{CD}$ is obtained when the PU is inactive $(\theta=0)$. The $\mathrm{CD}$ applies the maximal-ratio combining (MRC) for the received signals from the CS in the phase $T_{1}$ and the CR in the phase $T_{2}$. Using Eqs. (2) and (4), the CSNR at the $\mathrm{CD}$ is obtained as: 


$$
\gamma_{0}=\frac{G_{S D} P_{S}}{P_{U}}+\frac{\beta G_{R D} G_{S R} P_{S}}{\beta G_{R D} P_{U}+P_{U}} .
$$

The transmission power of the $\mathrm{CR}$ can be expressed as:

$$
P_{R}=\beta\left(G_{S R} P_{S}+\theta G_{P R} P_{P}+P_{U}\right) .
$$

Thus, if $\alpha$ is the probability that the PU is active, then the average transmission power of the $\mathrm{CR}$ is given by:

$$
\bar{P}_{R}=\alpha P_{R}(\theta=1)+(1-\alpha) P_{R}(\theta=0)=\beta\left(G_{S R} P_{S}+\alpha G_{P R} P_{P}+P_{U}\right) .
$$

We considered that the CRN has a constant total transmission power (i.e., the sum of the transmission powers of the $\mathrm{CS}$ and the $\mathrm{CR}$ is a constant, let it to be $P_{\max }$ ). The optimum power allocation is then:

$$
P_{S}+\bar{P}_{R} \leq P_{\max }
$$

Using Eq. (11), with the power equality constraint, Eq. (12) can be rewritten as:

$$
P_{S}+\bar{P}_{R}=P_{S}+\beta\left(G_{S R} P_{S}+\alpha G_{P R} P_{P}+P_{U}\right)=P_{\max } .
$$

Substituting the value of $P_{S}$ from Eq. (13) into Eq. (9) for the expression of the received CSNR, we obtain:

$$
\gamma_{0}=\left(G S D+\frac{\beta G_{R D} G_{S R}}{1+\beta G_{R D}}\right) \times \frac{\gamma_{\max }-\beta\left(1+\alpha \gamma_{P R}\right)}{1+\beta G_{S R}},
$$

where $\gamma_{\max }=P_{\max } / P_{U}$ and $\gamma_{P R}=G_{P R} P_{P} / P_{U}$ is the SNR of the signal transmitted over the link PU $\rightarrow$ CR.

\subsection{Average Probability of Symbol Error of the CR}

In this subsection we describe the evaluation of the average SER for the case of different distributions of the fading channel. For the evaluation of the average probability of the SER, we considered two types of fading channels - the Rayleigh and Nakagami- $m$ fading channels - for the amplitude of the transmitted signals (signals transmitted from the CS and CR to the CD) of the CRN network. At the CD, the combined signal is obtained from the MRC of the signals of the links CS to $\mathrm{CD}$ in phase $T_{1}$, and $\mathrm{CR}$ to $\mathrm{CD}$ in phase $T_{2}$. The sum of the two SNRs, $\gamma_{0}=\gamma_{S D}+\gamma_{R D}$ are the squares of the amplitudes of the signals. Therefore, the CSNR is then distributed according to the chi-squared and gamma distribution for the amplitude distribution in the Rayleigh and Nakagami- $m$ fading channels, respectively. The probability density functions (PDFs) of the CSNR are then as follows [14]: 


$$
f_{C h i-S q}(\gamma, \beta)=\frac{1}{(m-1) ! \gamma_{0}^{m}(\beta)} e^{-\gamma / \gamma_{0}(\beta)}
$$

and

$$
f_{\text {Gamma }}(\gamma, \beta)=\frac{m^{m} \gamma^{m-1}}{\gamma_{0}^{m}(\beta) \Gamma(m)} e^{-m \gamma / \gamma_{0}(\beta)},
$$

where $m$ is the number of the combiner paths at the CD. It is to be noted here that we have $m=2$. The average probability of the symbol error is calculated according to the following formula [15]:

$$
P_{S E R}(\beta)=\int_{0}^{\infty} P_{e}(\mathrm{e} \mid \gamma) f_{X}(\gamma, \beta) d \gamma
$$

where $X$ stands for the appropriate PDF and $P_{e}(\mathrm{e} \mid \gamma)$ represents the conditional symbol error probability for specific modulation technique for given SNR $\gamma$. In the next section, we plot the probability of SER against the power-amplifying factor $\beta$.

\subsection{Throughput of the CRN}

The conditional probability, $P\left(\mathcal{H}_{1} \mid \mathcal{H}_{0,-}\right)$ for the given sub-state $\mathcal{H}_{0,-}$ denotes the CFA probability, which is shown to be:

$$
P\left(\mathcal{H}_{1} \mid \mathcal{H}_{0,-}\right)=Q\left(\frac{\lambda-E\left(Y_{0}\right)}{\sqrt{D\left(Y_{0}\right)}}\right) \equiv Q\left(\frac{\lambda-N \mu_{0}(\beta)}{\sqrt{N} \mu_{0}(\beta)}\right)
$$

The detection probability is:

$$
p_{D}(r)=Q\left(\frac{\lambda-E\left(Y_{1}\right)}{\sqrt{D\left(Y_{1}\right)}}\right) \equiv Q\left(\frac{\lambda-N \mu_{1}(\beta, r)}{\sqrt{N} \mu_{1}(\beta, r)}\right) .
$$

The conditional probability $P\left(\mathcal{H}_{1} \mid \mathcal{H}_{0,+}\right)$ for the given sub-state $\mathcal{H}_{0,+}$, is known as the SFA probability and its expression is given by [9]:

$$
\begin{aligned}
P\left(\mathcal{H}_{1} \mid \mathcal{H}_{0,+}\right) & =1-\int_{r_{s}}^{R_{0}}\left(1-p_{D}(r)\right) f(r) d r \\
& =1-\int_{r_{s}}^{R_{0}}\left(1-Q\left(\frac{\lambda-N \mu_{1}(\beta, r)}{\sqrt{N} \mu_{1}(\beta, r)}\right)\right) f(r) d r,
\end{aligned}
$$

where $f(r)$ is the PDF of the PU location and is given by $f(r)=2 r / R_{0}^{2}$ as $f(r)$ should give 
the normalization condition of: $\int_{0}^{R_{0}} f(r) d r \equiv 1$.

The total medium access probability $P_{M A}(\beta)$ is given by the following expression:

$$
P_{M A}=(1-\alpha)\left[1-P\left(\mathcal{H}_{1} \mid \mathcal{H}_{0,-}\right)\right]+\alpha\left[1-P\left(\mathcal{H}_{1} \mid \mathcal{H}_{0,+}\right)\right] \text {. }
$$

After substituting Eqs. (18) and (20) for the expressions of $P\left(\mathcal{H}_{1} \mid \mathcal{H}_{0,-}\right)$ and $P\left(\mathcal{H}_{1} \mid \mathcal{H}_{0,+}\right)$ into the above equation for the expression of the total medium access probability, Eq. (21) becomes:

$$
P_{M A}(\beta)=(1-\alpha)\left[1-Q\left(\frac{\lambda-N \mu_{0}(\beta)}{\sqrt{N} \mu_{0}(\beta)}\right)\right]+\alpha\left[\frac{2}{R^{2}} \int_{r_{s}}^{R}\left(1-Q\left(\frac{\lambda-N \mu_{1}(\beta, r)}{\sqrt{N} \mu_{1}(\beta, r)}\right)\right) r d r\right]
$$

The throughput of the secondary network is then defined by [8]:

$$
R(\beta)=\frac{1}{2} P_{M A}(\beta) \log _{2}\left[1+\gamma_{0}(\beta)\right]
$$

which is measured in the unit of bits per second per $\mathrm{Hz}(\mathrm{bps} / \mathrm{Hz})$. It is to be mentioned here that in calculating the secondary throughput $R$ given by Eq. (23), the authors of [8] only considered the first term of Eq. (21) for the total medium access probability $P_{M A}$. However, for actual quantification of the secondary medium access probability, the second term of Eq. (21) also has to be considered along with the first term in calculating the secondary throughput of the cognitive network. The first term of the expression of $P_{M A}$ in Eq. (21) is due to the CFA, while the second term is due to the effect of the SFA probability, about which no importance is given in the current literature.

In the next section we plot the throughput $R$, against the amplifying factor $\beta$, under the power constraint of the CRN network including both the CFA and the combination of CFA and SFA probabilities.

\section{RESULtS}

This section deals with the profile of the SER and the throughput against the power amplification factor $\beta$. We take the numerical values of different parameters as [8]: $N=1000, \alpha=0.3$ (which means PU is present for $30 \%$ of the total time), $P_{u}=0.93 \mathrm{dBW}$ and $P_{p}=10 \mathrm{dBW}$. Here a specific channel case is considered:

$$
G_{s r}=G_{r d}=G_{p r}=-4 \mathrm{~dB}, G_{s d}=G_{p s}=-10 \mathrm{~dB}, \quad \gamma_{p s}=-10 \mathrm{~dB}, \gamma_{p r}=-4 \mathrm{~dB}
$$

The probability of the average SER is plotted against the power amplification factor $\beta$ in Fig. 2 for the QPSK modulation scheme and in Fig. 3 for the 8-PSK modulation scheme under the Rayleigh and Nakagami- $m$ fading channels. It is visualized from both the figures that the SER is much higher in the Nakagami- $m$ fading case, as compared to the Rayleigh fading. It is interesting to observe from the figures that the SER is minimum at a particular value of the 
power amplification factor $\beta$. It is also observed that as $\beta$ increases, the SER also increases after attaining the minimum value. It is further noticed from these two figures that the SER is

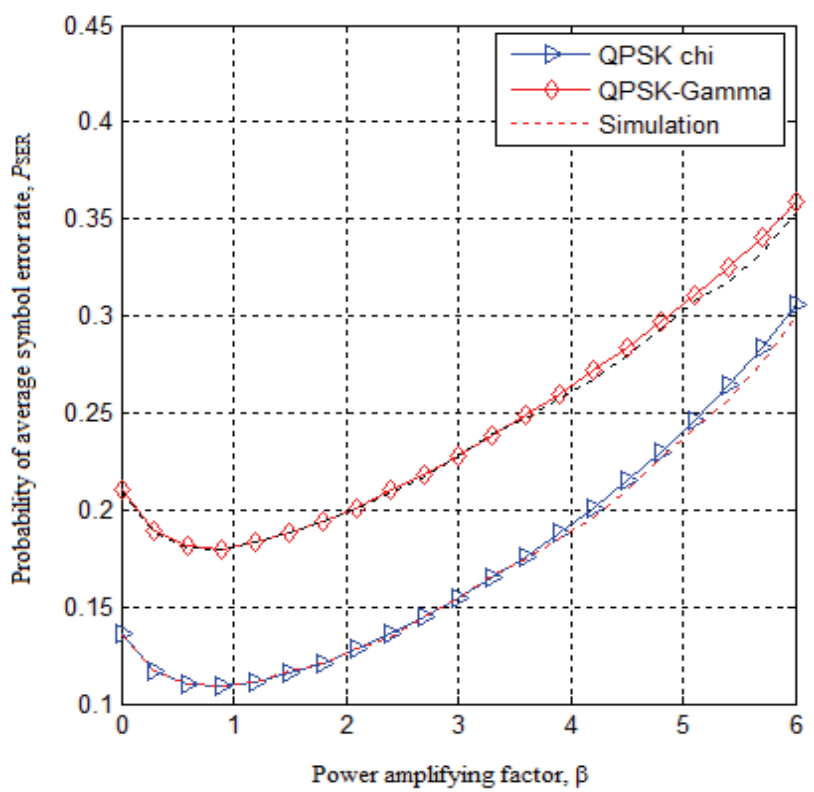

Fig. 2. Probability of the average symbol error rate, $P_{S E R}$, as a function of the power amplifying factor $\beta$, of the cognitive relay.

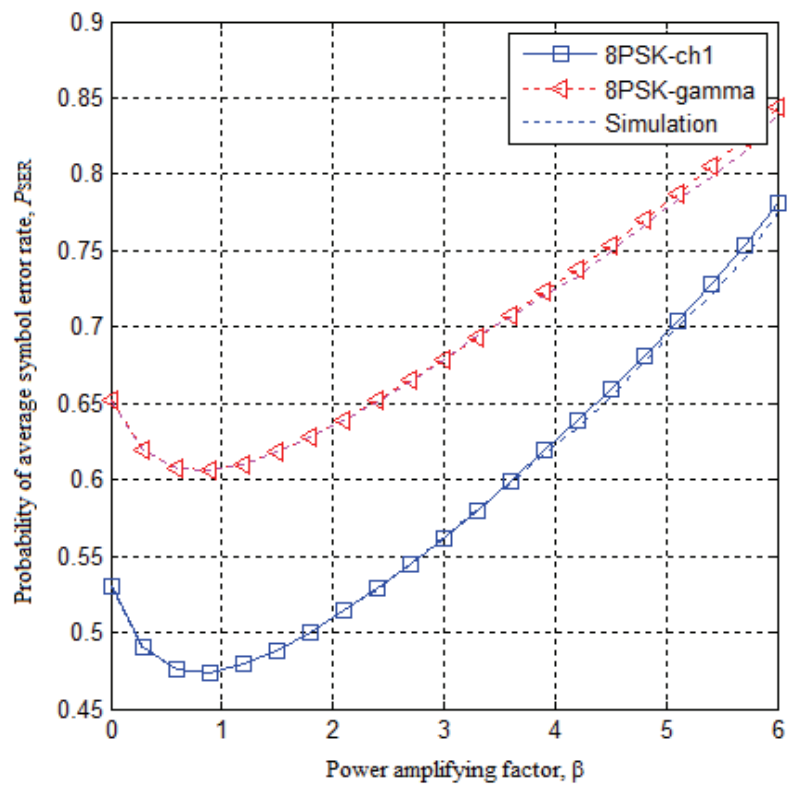

Fig. 3. Probability of the average symbol error rate, $P_{S E R}$, as a function of the power amplifying factor $\beta$, of the cognitive relay. 


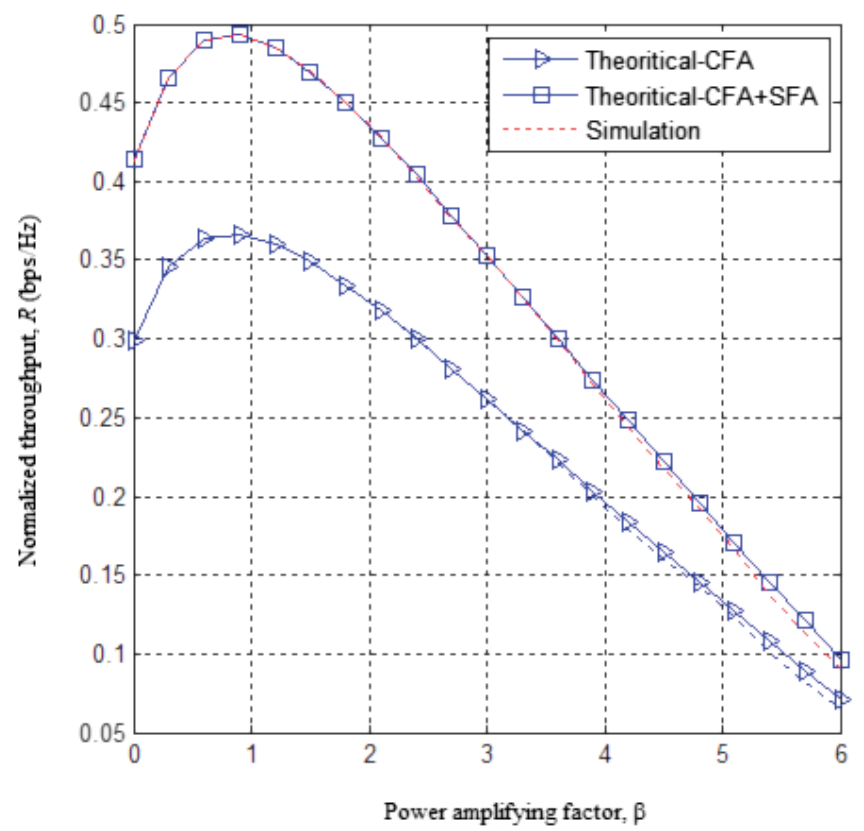

Fig. 4. Secondary throughput $R$, as a function of the power amplifying factor $\beta$, of the cognitive relay. $C F A=$ conventional false alarm, SFA=spatial false alarm.

much higher in the case of the 8-PSK modulation scheme, as compared to the QPSK modulation. Hence, our relay model works better under a Rayleigh fading environment with QPSK modulation. Monte-Carlo simulation results are also shown in the figures as dotted curves. It is observed that the theoretical curves coincide with the simulation results and have a slight deviation at the higher values of the power amplification factor $\beta$.

Taking similar parameters, the throughput of the CRN is plotted against the power amplification factor $\beta$ in Fig. 4. Considering only the CFA and the combination of CFA and SFA, the throughput is found to be much higher using the combination of CFA and SFA, as is visualized in Fig. 4. Without an incorporation of SFA, sometimes conflicts take place between the PU and the SU, which considerably reduces the throughput. By the incorporation of SFA, the above conflict is alleviated to a certain extent, and the throughput is enhanced. Both the curves of the throughput have a maxima at the same value of the power amplification factor $\beta$, which yields the minimum average SER found in Figs. 2 and 3. We also ran a Monte-Carlo simulation in calculating the throughput to validate the theoretical results.

\section{Conclusions}

This paper provides a non-linear relationship between the power amplification factor $\beta$, and the throughput $R$, of the CRN. At the same time, it shows another nonlinear relation between the power amplification factor $\beta$, and the probability of average SER. The system operates at an optimum position for a particular value of the power amplification factor $\beta$, which maximizes the throughput $R$, and minimizes the probability of the average SER, which is the central idea of this paper. It is further shown that the inclusion of SFA in the analysis considerably enhances 
the throughput of the secondary network, as compared to the case when only the CFA is considered. By increasing the number of antennas at the CS, cognitive relay, and cognitive destination, we can apply different combinations of multiple input multiple outputs (MIMOs), multiple input single output (MISOs), single input multiple outputs (SIMO), and the MRC scheme to observe changes in the performance of the network. Incorporation of the adaptive equalizer at the relay or destination node can further enhance the performance of the network.

\section{REFERENCES}

[1] I. F. Akyildiz, W.Y. Lee, and K. R. Chowdhury, "CRAHNs: cognitive radio ad hoc networks," $A d$ Hoc Networks, vol. 7, no. 5, pp. 810-836, Jul. 2009.

[2] S. Haykin, "Cognitive radio: brain-empowered wireless communications," IEEE Journal on Selected Areas in Communications, vol. 23, no. 2, pp. 201-220, Feb. 2005.

[3] I. F. Akyildiz, W.Y. Lee, M. C. Vuran, and S. Mohanty, "NeXt generation/dynamic spectrum access/cognitive radio wireless networks: a survey," Computer Networks, vol. 50, no. 13, pp. 21272159, Sep. 2006.

[4] S. Haykin, D. J. Thomson, and J. H. Reed, "Spectrum sensing for cognitive radio," Proceedings of the IEEE, vol. 97, no. 5, pp. 849-877, May 2009.

[5] J. Mitola III, "Cognitive radio: an integrated agent architecture for software defined radio," Ph.D. dissertation, Royal Institute of Technology (KTH), Stockholm, Sweden, 2000.

[6] J. Mitola III and G. Q. Maguire Jr., "Cognitive radio: making software radios more personal," IEEE Personal Communications, vol. 6, no. 4, pp. 13-18, Aug. 1999.

[7] T. Yucek and H. Arslan, "A survey of spectrum sensing algorithms for cognitive radio applications," IEEE Communications Surveys \& Tutorials, vol. 11, no. 1, pp. 116-130, Mar. 2009.

[8] S. Huang, H. Chen, and Y. Zhang, "Optimal power allocation for spectrum sensing and data transmission in cognitive relay networks," IEEE Wireless Communications Letters, vol. 1, no. 1, pp. 26-29, Feb. 2012.

[9] W. Han, J. Li, Q. Liu, and L. Zhao, "Spatial false alarms in cognitive radio," IEEE Communications Letters, vol. 15, no. 5, pp. 518-520, May 2011.

[10] Y. C. Liang, Y. Zeng, E. C. Y. Peh, and A. T. Hoang, "Sensing-throughput tradeoff for cognitive radio networks," IEEE Transactions on Wireless Communications, vol. 7, no. 4, pp. 1326-1337, Apr. 2008.

[11] A. Ghasemi and E. S. Sousa, "Optimization of spectrum sensing for opportunistic spectrum access in cognitive radio networks," in Proceedings of the 4th IEEE Consumer Communications and Networking Conference, Las Vegas, NV, January 11-13, 2007, pp. 1022-1026.

[12] J. Shen, T. Jiang, S. Liu, and Z. Zhang, "Maximum channel throughput via cooperative spectrum sensing in cognitive radio networks," IEEE Transactions on Wireless Communications, vol. 8, no. 10, pp. 5166-5175, Oct. 2009.

[13] E. Peh and Y. C. Liang, "Optimization for cooperative sensing in cognitive radio networks," in Proceedings of the IEEE Wireless Communications and Networking Conference, Kowloon, Hong Kong, March 11-15, 2007, pp. 27-32.

[14] M. K. Simon and M.S. Alouini, Digital Communication Over Fading Channels: A Unified Approach to Performance Analysis. New York, NY: John Wiley \& Sons, 2000.

[15] S. S. Haykin and M. Moher, Modern Wireless Communications. Upper Saddle River, NJ: Pearson/Prentice Hall, 2005. 


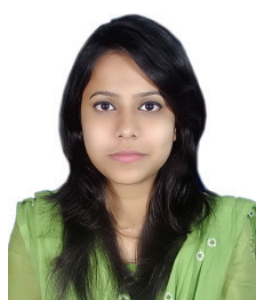

\section{Tasnina A. Tishita}

She has completed her B.Sc. degree in Electronic and Telecommunication Engineering under the Department of Electronics and Communications Engineering at East West University, Dhaka, Bangladesh in 2013. Now she is working as a teaching assistant in the same Department.

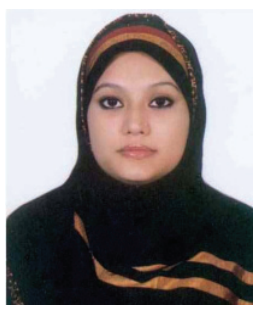

\section{Sumiya Akhter}

She has completed her B.Sc. degree in Electronic and Telecommunication Engineering under the Department of Electronics and Communications Engineering at East West University, Dhaka, Bangladesh in 2013.

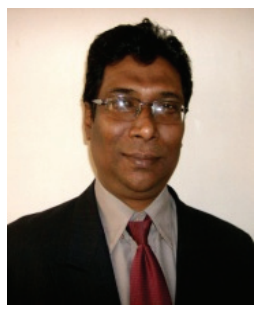

\section{Md. Imdadul Islam}

He has completed his B.Sc. and M.Sc. Engineering in Electrical and Electronic Engineering from Bangladesh University of Engineering and Technology, Dhaka, Bangladesh in 1993 and 1998, respectively and has completed his Ph.D. degree from the Department of Computer Science and Engineering, Jahangirnagar University, Dhaka, Bangladesh in the field of network traffic engineering. He is now working as a Professor at the Department of Computer Science and Engineering, Jahangirnagar University, Savar, Dhaka, Bangladesh. Previously, he worked as an Assistant Engineer in Sheba Telecom (Pvt.) LTD (A joint venture company between Bangladesh and Malaysia, for Mobile cellular and WLL), from Sept'94 to July'96. He has a very good field experience in installation of Radio Base Stations and Switching Centers for WLL. His research field is network traffic, wireless communications, wavelet transform, OFDMA, WCDMA, adaptive filter theory, ANFIS and array antenna systems. He has more than hundred research papers in national and international journals and conference proceedings.

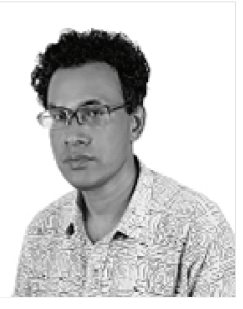

\section{R. Amin}

He has received his B.S. and M.S. degrees in Physics from Jahangirnagar University, Dhaka, Bangladesh in 1984 and 1986 respectively and his Ph.D. degree in Plasma Physics from the University of St. Andrews, UK in 1990. He is a Professor of Electronics and Communications Engineering at East West University, Dhaka, Bangladesh. He served as a Post-Doctoral Research Associate in Electrical Engineering at the University of Alberta, Canada, during 1991-1993. He was an Alexander von Humboldt Research Fellow at the Max-Planck Institute for Extraterrestrial Physics at Garching/Munich, Germany during 1997-1999. Dr. Amin awarded the Commonwealth Postdoctoral Fellowship in 1997. Besides these, he has also received several awards for his research, including the Bangladesh Academy of Science Young Scientist Award for the year 1996 and the University Grants Commission Young Scientist Award for 1996. He is a member of the IEEE. His current research is in the broad field of wireless communications. 